\title{
Identification of an Intersubunit Cross-Link between Substituted Cysteine Residues Located in the Putative ATP Binding Site of the P2X $\mathrm{X}_{1}$ Receptor
}

\author{
Benjamin Marquez-Klaka, ${ }^{1}$ Jürgen Rettinger, ${ }^{2}$ Yogesh Bhargava, ${ }^{2}$ Thomas Eisele, ${ }^{3}$ and Annette Nicke ${ }^{1}$ \\ ${ }^{1}$ Department of Neurochemistry, Max-Planck-Institute for Brain Research, D-60528 Frankfurt, Germany, ${ }^{2}$ Department of Biophysical Chemistry, \\ Max-Planck-Institute of Biophysics, D-60438 Frankfurt, Germany, and ${ }^{3}$ Department of Molecular Pharmacology, RWTH Aachen University, D-52074 \\ Aachen, Germany
}

\begin{abstract}
P2X receptors are ATP-gated nonselective cation channels. Functional receptors are assembled as homotrimers or heterotrimers of seven cloned subunits. Each subunit contains two transmembrane domains linked by a large extracellular loop that is required for agonist binding. So far, there is no direct evidence indicating whether the agonist binding site is formed within one subunit or at the interface of two neighboring subunits. Here we used a disulfide cross-linking approach to identify pairs of residues that are in close proximity within the ATP binding site of the $\mathrm{P} 2 \mathrm{X}_{1}$ homotrimer. Eight amino acid residues that have previously been shown to be essential for high ATP potency (K68, K70, F185, K190, F291, R292, R305, and K309) were substituted by cysteine residues, and the respective mutant subunits were pairwise expressed in Xenopus laevis oocytes. Nonreducing SDS-PAGE analysis of the purified receptors revealed a spontaneous and specific dimer formation between the K68C and F291C mutants. An almost complete cross-link into trimers was achieved with the K68C/F291C double mutant, consistent with the formation of intersubunit disulfide bridges. In support of this interpretation, twoelectrode voltage-clamp analysis of the K68C/F291C mutations introduced into a nondesensitizing P2X $\mathrm{X}_{2-1}$ chimera showed only small ATP-activated currents that, however, increased $\sim 60$-fold after extracellular application of the reducing agent dithiothreitol. In addition, we show that a $\mathrm{K} 68 \mathrm{C} / \mathrm{K} 309 \mathrm{C}$ double mutant is nonfunctional and can be functionally rescued by coexpression with nonmutated subunits. Our data are consistent with loops from neighboring P2X subunits forming the ATP-binding site in P2X receptors.
\end{abstract}

Key words: ATP binding site; P2X receptor; disulfide cross-linking; subunit interface; cysteine substitution; two-electrode voltage-clamp

\section{Introduction}

ATP, acting on $\mathrm{P} 2 \mathrm{X}$ receptors ( $\mathrm{P} 2 \mathrm{XRs}$ ), has been shown to function as both a modulator of neurotransmission and a principal neurotransmitter (Finger et al., 2005). P2XRs thus represent, in addition to the ionotropic glutamate receptors (iGluRs) and the Cys-loop receptors of the nicotinic acetylcholine receptor (nAChR) superfamily, a third family of neurotransmitter-gated ion channels. A single P2X subunit contains two transmembrane domains linked by a large extracellular loop (North, 2002). The trimeric assembly of these subunits (Nicke et al., 1998) has been confirmed by atomic force microscopy (Barrera et al., 2005).

For both nAChRs and iGluRs, soluble homologous proteins have been identified that represent valuable templates for homology modeling of their ligand-binding sites (O'Hara et al., 1993; Brejc et al., 2001). The ACh-binding site is formed by the extracellular N-terminal domain of the pentameric nAChRs and has been localized at the interface of neighboring nAChR subunits,

Received July 21, 2006; revised Dec. 5, 2006; accepted Dec. 31, 2006.

This work was supported by Deutsche Forschungsgemeinschaft Grant NI 592/3. We thank Heinrich Betz for generous support and critical reading of this manuscript and Eva-Verena Bongartz for technical help.

Correspondence should be addressed to Dr. Annette Nicke, Max-Planck-Institute for Brain Research, Department of Neurochemistry, Deutschordenstrasse 46, D-60528 Frankfurt, Germany. E-mail: anicke@gwdg.de.

DOI:10.1523/JNEUROSCI.3105-06.2007

Copyright $\odot 2007$ Society for Neuroscience $\quad$ 0270-6474/07/271456-11\$15.00/0 with aromatic residues from both subunits contributing to ligand binding. In the case of tetrameric iGluRs, the agonist binding site is formed by two segments (extracellular $\mathrm{N}$ terminus and the loop between TM3 and TM4) of a single subunit that are separated by two transmembrane domains and a reentry loop. No homology model exists for P2XRs, and they do not contain consensus motifs common to other ATP-binding proteins. Thus, the location of the ATP-binding site(s) and the structural rearrangements that lead to channel opening are still unclear.

On the basis of secondary structure predictions, it has been proposed that the second half of the extracellular loop of P2XRs might be folded in $\beta$-sheets (Freist et al., 1998), similar to the catalytic domains of class II aminoacyl t-RNA synthetases. With this model as a template for rational mutagenesis, residues K190, $\mathrm{F} 230, \mathrm{R} 278$, and $\mathrm{D} 280$ of the $\mathrm{P} 2 \mathrm{X}_{4} \mathrm{R}$ were proposed to contribute to ATP binding and shown to reduce ATP potency $>1000$-fold when mutated into alanine (Yan et al., 2005). Because t-RNA synthetases bind ATP within single subunits, these findings would suggest an intrasubunit localization of the ATP-binding site. Systematic substitution of conserved amino acid residues revealed that positively charged residues exofacial to the first and second proposed transmembrane domains (K68, K70, R292, and $\mathrm{K} 309$ in $\mathrm{P} 2 \mathrm{X}_{1}$ and the corresponding residues in $\mathrm{P} 2 \mathrm{X}_{2}$ ) are crucial for ATP potency, presumably by interacting with the negatively 
charged phosphate chain of ATP (Ennion et al., 2000; Jiang et al., 2000; Roberts und Evans, 2004); however, in a recent study on the $\mathrm{P}_{2} \mathrm{X}_{4}$ subtype, a more indirect function of K309 in ATP binding has been suggested (Yan et al., 2006). The location of these lysine residues on opposite ends of the extracellular loop opens the possibility of an intersubunit ATP binding site (Nagaya et al., 2005; Wilkinson et al., 2006). In addition, the mutation of conserved aromatic and polar amino acid residues (F185, T186, N290, and F291) influences ATP potency, and hence these residues have been suggested to coordinate the adenine ring (Roberts and Evans, 2004).

In this study, we used site-directed disulfide mapping to investigate the spatial arrangements of residues believed to contribute to ATP binding and thereby to determine the localization of the ATP-binding pocket.

\section{Materials and Methods}

cDNA constructs and cRNA synthesis. Construction of N-terminal hexahistidyl-tagged rat $\mathrm{P} 2 \mathrm{X}_{1}\left(\right.$ His- $\left.\mathrm{P} 2 \mathrm{X}_{1}\right)$ and chimeric rat $\mathrm{P} 2 \mathrm{X}_{2-1}$ (His$\mathrm{P} 2 \mathrm{X}_{2-1}$ ) cDNAs in the pNKS2 oocyte expression vector has been described previously (Nicke et al., 1998; Rettinger and Schmalzing, 2004). Site-directed mutagenesis was performed with the QuickChange mutagenesis kit (Stratagene, La Jolla, CA), and primers were synthesized by MWG Biotech (Ebersberg, Germany). Sequences were verified by didesoxynucleotide sequencing (MWG Biotech). Capped cRNA was synthesized from linearized templates with SP6 RNA polymerase by using the Message Machine kit (Ambion, Austin, TX).

Oocyte injection and protein labeling. Xenopus laevis toads were obtained from Nasco International (Fort Atkinson, WI). Oocytes were prepared as described previously (Nicke et al., 1998) and injected with $50 \mathrm{nl}$ aliquots of cRNA $(0.5 \mu \mathrm{g} / \mu \mathrm{l})$. To obtain similar amounts of both mutants in coexpression experiments, cRNA ratios were adjusted to account for the different expression levels of the mutants. cRNA-injected oocytes and noninjected controls were metabolically labeled by overnight incubation at $19^{\circ} \mathrm{C}$ with L- $\left[{ }^{35} \mathrm{~S}\right]$ methionine at $\sim 40 \mathrm{MBq} / \mathrm{ml}$ with $\sim 0.1 \mathrm{MBq}$ per oocyte (PerkinElmer Life and Analytical Sciences, RodgauJügesheim, Germany) in sterile oocyte solution containing (in mM): 96 $\mathrm{NaCl}, 2 \mathrm{KCl}, 1 \mathrm{CaCl}_{2}, 1 \mathrm{MgCl}_{2}$, and 5 HEPES, pH 7.4, supplemented with gentamycin (50 $\mu \mathrm{g} / \mathrm{ml}$; Sigma, Taufkirchen, Germany), and subsequently kept for $24-48 \mathrm{~h}$ in $10 \mathrm{~mm}$ unlabeled methionine in the above oocyte solution. For selective labeling of plasma membrane proteins, intact oocytes were selected 3-4 d after injection and incubated for 30 min at $4^{\circ} \mathrm{C}$ in $50 \mu \mathrm{M}$ Cy5-conjugated $N$-hydroxysuccinimide (NHS)ester (GE Healthcare, Munich, Germany) in labeling buffer $(110 \mathrm{~mm}$ $\mathrm{NaCl}, 0.5 \mathrm{~mm} \mathrm{CaCl}_{2}, 20 \mathrm{~mm}$ sodium phosphate buffer, $\mathrm{pH}$ 8.5). Oocytes were subsequently washed and extracted as described below.

Purification of hexahistidyl-tagged proteins. Protein complexes containing His-tagged P2X subunits were purified under nondenaturing conditions from digitonin extracts of $X$. laevis oocytes as described previously (Nicke et al., 1998). Briefly, oocytes were homogenized in $0.1 \mathrm{M}$ sodium phosphate buffer, $\mathrm{pH} 8.0$ (20 $\mu$ l per oocyte) containing $0.4 \mathrm{~mm}$ Pefabloc SC (Fluka, Buchs, Switzerland) and 1\% (w/v) digitonin (Fluka). The homogenate was incubated on ice for $15 \mathrm{~min}$, and the extract was then cleared by centrifugation (10 min at $15,000 \mathrm{rpm}$ in a desktop centrifuge). Then $100 \mu \mathrm{l}$ of the clear supernatant were diluted with $400 \mu \mathrm{l}$ of the above buffer and supplemented with $30 \mu \mathrm{l}$ of $\mathrm{Ni}^{2+}$-nitrilotriacetic acid agarose (NTA) agarose beads (Qiagen, Hilden, Germany) and 10 $\mathrm{mm}$ imidazole. After $30 \mathrm{~min}$ of incubation under continuous inversion at $4^{\circ} \mathrm{C}$, the agarose-bound protein was washed four times with $1 \mathrm{ml}$ of phosphate buffer containing $0.2 \%$ (w/v) digitonin, $0.4 \mathrm{~mm}$ Pefabloc SC, and $30 \mathrm{~mm}$ imidazole. Subsequently, protein was eluted from the agarose beads with nondenaturing elution buffer [20 mM Tris- $\mathrm{HCl}, 100 \mathrm{~mm}$ imidazole-HCl, 10 mm EDTA, 1\% (w/v) digitonin, pH 7.8]. Eluted protein was kept at $0^{\circ} \mathrm{C}$ until analyzed.

Blue native PAGE and SDS-PAGE. Blue native PAGE (BN-PAGE) was performed as described previously (Schägger et al., 1994; Nicke et al., 1998). Purified protein samples were supplemented with blue native sample buffer to a final concentration of $10 \%(\mathrm{w} / \mathrm{v})$ glycerol, $0.2 \%(\mathrm{w} / \mathrm{v})$ Serva blue G (Serva, Heidelberg, Germany), and $20 \mathrm{~mm}$ sodium 6 -amino- $n$-caproate, and resolved on $4-13 \%$ polyacrylamide gradient gels flanked by two lanes of molecular mass markers (GE Healthcare) at the sides of the gel. For SDS-PAGE, protein samples were supplemented with nonreducing SDS sample buffer and run in parallel with molecular mass markers (Precision Plus Protein All Blue Standard; Bio-Rad, Munich, Germany). Radioactive gels were dried and exposed to BioMax MR films (Kodak, Stuttgart, Germany) at $-80^{\circ} \mathrm{C}$ or to a phosphor-imaging plate (for quantification purposes). Fluorescent gels and phosphor plates were scanned on a Typhoon Trio fluorescence scanner and analyzed with Image Quant TL software (GE Healthcare). Experiments were repeated at least three times.

Glycosylation analysis. For analysis of the glycosylation status, $10 \mu \mathrm{l}$ aliquots of purified protein were supplemented with reducing $[20 \mathrm{~mm}$ dithiothreitol (DTT)] SDS sample buffer and 1\% (w/v) octylglucoside (Calbiochem, La Jolla, CA), and incubated for $2 \mathrm{~h}$ at $37^{\circ} \mathrm{C}$ with $5 \mathrm{IUB}$ milliunits of endoglycosidase $\mathrm{H}$ (EndoH) or PNGase F (peptide: $N$-glycosidase F; New England Biolabs, Frankfurt, Germany) as indicated.

Reduction and oxidization of P2XR complexes in the presence and absence of ATP. Protein extracts were prepared, and His-tagged protein complexes were bound to $\mathrm{Ni}^{2+}$-NTA agarose beads as described above (see Purification of hexahistidyl-tagged proteins). After two washing steps, the beads were split into two aliquots and incubated for $30 \mathrm{~min}$ under constant shaking at $4^{\circ} \mathrm{C}$ in washing buffer supplemented with 20 mм $\beta$-mercaptoethanol. Subsequently, the two fractions were washed (three times) and eluted either in the presence or absence of the indicated concentrations of ATP or, as an alternative control, with $10 \mathrm{~mm}$ ADP in the washing and elution buffer. These ATP-protected and nonprotected preparations were resolved by SDS-PAGE directly (control for effective reduction by $\beta$-mercaptoethanol) and after oxidation by the addition of $2 \mathrm{mM} \mathrm{H}_{2} \mathrm{O}_{2}$.

Electrophysiological recordings. Current responses to ATP were measured 1-3 d after cRNA injection into $X$. laevis oocytes by using the two-electrode voltage-clamp technique. The amount of injected cRNA $(0.05$ or $0.5 \mu \mathrm{g} / \mu \mathrm{l})$ and the expression time in functional experiments were determined individually for each construct to avoid current amplitudes that were too large. The standard solution used to superfuse the oocytes contained (in mM): $90 \mathrm{NaCl}, 1 \mathrm{KCl}, 2 \mathrm{MgCl}_{2}$, and $5 \mathrm{HEPES}, \mathrm{pH}$ $7.4\left(\mathrm{Mg}^{2+}\right.$ oocyte Ringer's solution). Calcium salts were omitted to avoid activation of endogenous $\mathrm{Ca}^{2+}$-activated $\mathrm{Cl}^{-}$channels after the opening of P2XRs. Glass microelectrodes were filled with $3 \mathrm{~m} \mathrm{KCl}$ solution and had resistances $<1 \mathrm{M} \Omega$. Two bath electrodes were used to minimize series resistance errors. In experiments during which reducing or oxidizing compounds were applied, the reference electrode for voltage recording was connected to the bath via a $3 \mathrm{M} \mathrm{KCl} /$ agar bridge to avoid voltage errors. Currents were recorded with a TEC-03 or a TEC-05 amplifier (npi electronics, Lambrecht, Germany), low-pass filtered at $100 \mathrm{~Hz}$, and sampled at $200 \mathrm{~Hz}$. If not noted otherwise, the oocytes were voltage clamped at $-60 \mathrm{mV}$. A fast and reproducible solution exchange between different solutions was achieved by using a $\sim 50 \mu \mathrm{l}$ recording chamber perfused at a rate of $\sim 10 \mathrm{ml} / \mathrm{min}$ (Rettinger and Schmalzing, 2003). Solutions were switched by magnetic valves under the control of Cellworks software (npi electronics). All measurements were performed at room temperature $\left(20-22^{\circ} \mathrm{C}\right)$. Data are presented as mean \pm SE from $n$ experiments. $\mathrm{EC}_{50}$ values were calculated from a nonlinear fit of the Hill equation to the data with Origin software (OriginLab, Northampton, MA).

\section{Results}

On the basis of the available mutagenesis data for $\mathrm{P} 2 \mathrm{X}_{1} \mathrm{Rs}$ and $\mathrm{P} 2 \mathrm{X}_{2} \mathrm{Rs}$ (Table 1), we selected eight conserved aromatic or positively charged residues in the extracellular domain of the P2XR and substituted the corresponding residues in the hexahistidyltagged rat $\mathrm{P} 2 \mathrm{X}_{1}$ subunit by cysteine residues. The selected residues (indicated by star symbols) had been shown to considerably reduce ATP potency when mutated into alanine residues (Ennion et al., 2000; Jiang et al., 2000; Roberts and Evans, 2004) and are contributed to by four regions that we designated regions A-D 
Table 1. Summary of published $\mathrm{EC}_{50}$ values of $A T P$ at $\mathrm{P} 2 \mathrm{X}_{1}, \mathrm{P} 2 \mathrm{X}_{2}$, and $\mathrm{P} 2 \mathrm{X}_{4}$ receptors with alanine substitutions (and isoleucine in the case of $\mathrm{P} 2 \mathrm{X}_{2} \mathrm{~F} 271 \mathrm{I}$ ) of conserved residues and/or residues presumed to contribute to the ATP binding site

\begin{tabular}{|c|c|c|c|c|c|}
\hline Corresponding residues in $\mathrm{P} 2 \mathrm{X}_{1}, \mathrm{P} 2 \mathrm{X}_{2}$, and $\mathrm{P} 2 \mathrm{X}_{4}$ & $\mathrm{P} 2 \mathrm{X}_{1}$ & $\mathrm{P} 2 \mathrm{X}_{2}$ & ${\mathrm{P} 2 \mathrm{X}_{4}}$ & Binding of & Region \\
\hline wt & $\sim 1 \mu \mathrm{M}^{a, b}$ & $\sim 10 \mu \mathrm{M}^{\mathrm{c}}$ & $\sim 3 \mu \mathrm{m}^{d}$ & & \\
\hline${ }^{*} K 68 \mathrm{~A}, \mathrm{~K} 69 \mathrm{~A}, \mathrm{~K} 67 \mathrm{~A}$ & $\sim 1.4 \mathrm{~mm}^{a}$ & n.f..$^{c}$ & n.f. $e^{r}$ & Phosphate $^{b}$ & $A$ \\
\hline${ }^{*} \mathrm{~K} 70 \mathrm{~A}, \mathrm{~K} 71 \mathrm{~A}, \mathrm{~K} 69 \mathrm{~A}$ & $\sim 4 \mu \mathrm{M}^{a}$ & $>1000 \mu \mathrm{M}^{c}$ & n.d. & & A \\
\hline *F185A, F183A, F185A & $\sim 9 \mu \mathrm{m}^{b}$ & n.d. & n.d. & Adenine $^{b}$ & B \\
\hline *K190A, K188A, K190A & $\sim 4.3 \mu \mathrm{m}^{a}$ & $>1000 \mu \mathrm{m}^{c}$ & $>5000 \mu \mathrm{m}^{d}$ & Phosphate $^{d}$ & B \\
\hline F230A, F227I, F230A & $\sim 1.3 \mu \mathrm{M}^{b}$ & n.f. $^{f}$ & $>5000 \mu \mathrm{m}^{d}$ & Adenine $^{d}$ & \\
\hline G278A, R275A, R278A & n.d. & n.d. & $>5000 \mu \mathrm{m}^{d}$ & Phosphate $^{d}$ & \\
\hline Y280A, D277A, D280A & n.d. & n.d. & $>5000 \mu \mathrm{M}^{d}$ & $\mathrm{Mg}^{2+d}$ & \\
\hline N290A, N288A, N293A & $\sim 71 \mu \mathrm{M}^{g}$ & $\sim 1000 \mu \mathrm{M}^{\mathrm{c}}$ & n.d. & & (C) \\
\hline *F291A, F289A, F294A & $\sim 140 \mu \mathrm{M}^{b}$ & n.d. & n.d. & Adenine $^{b}$ & $C$ \\
\hline${ }^{*}$ R292A, R290A, R295A & $\sim 71 \mu \mathrm{M}^{a}$ & $>1000 \mu \mathrm{m}^{c}$ & n.d. & Adenine $^{b}$ & $C$ \\
\hline${ }^{*} \mathrm{R} 305 \mathrm{~A}, \mathrm{R} 304 \mathrm{~A}, \mathrm{R} 309 \mathrm{~A}$ & $\sim 3.7 \mu \mathrm{M}^{a}$ & $>1000 \mu \mathrm{M}^{c}$ & n.d. & & D \\
\hline *K309A, K308A, K313A & $\sim 1.1 \mathrm{~mm}^{a}$ & n.f. ${ }^{c}$ & $>3000 \mu \mathrm{m}^{h}$ & Phosphate $^{b}$ & $D$ \\
\hline
\end{tabular}

Asterisks indicate positions that were substituted by cysteine residues in this study. These selected residues were assigned to four regions ( $A-D)$ in which pairs of two to three relevant residues appeared to be clustered. $n$.d. and $n . f$. indicate mutations that were not investigated or not functional, respectively.

${ }^{a}$ Ennion, et al., 2000.

${ }^{b}$ Roberts and Evans, 2004 .

'Jiang et al., 2000.

¿Yan et al., 2005.

eWilkinson et al, 2006.

Nakazawa et al., 2004

${ }^{g}$ Roberts and Evans, 2006a.

${ }^{h}$ Yan et al., 2006

(Table 1). As a positive control for successful cross-linking, we chose the cysteine substitutions V48C and I329C. These residues are located at the extracellular ends of the first and second proposed transmembrane domain, respectively, and the corresponding residues in the $\mathrm{P} 2 \mathrm{X}_{2}$ and $\mathrm{P} 2 \mathrm{X}_{3}$ subunits have been functionally and biochemically shown to form an intersubunit disulfide cross-link (Jiang et al., 2001, 2003) that impedes channel gating rather than agonist binding.

\section{Validation of the correct folding and assembly of single cysteine mutants}

An important structural feature of all P2XRs are 10 conserved cysteine residues in the extracellular loop (see Fig. 6), and there is good evidence that all 10 cysteine residues form disulfide bridges that are critical for receptor structure and function (Clyne et al., 2002; Ennion and Evans, 2002). During overexpression in Xenopus oocytes, intersubunit cross-links between $\mathrm{P} 2 \mathrm{X}_{1}$ subunits have been observed after short chase periods, suggesting that an intermediate formation of intersubunit disulfide bridges and/or misfolding might occur during maturation of receptor complexes. In the mature receptors, only intrasubunit disulfide bridges are present because the complexes can be dissociated into monomers under nonreducing conditions (Nicke et al., 1998). To ensure that we were working with correctly folded and functional receptors, our mutations were done on $\mathrm{P}_{2} \mathrm{X}_{1} \mathrm{R}$ subunits with the background of all 10 cysteine residues. To further confirm that the additional cysteine residues did not unspecifically cross-link with themselves or any of the conserved cysteine residues, the metabolically labeled oocytes were chased for 2-3 d in nonradioactive methionine to allow for protein maturation. Digitoninsolubilized $\mathrm{P} 2 \mathrm{X}_{1} \mathrm{R}$ complexes were then isolated under nondenaturing conditions via $\mathrm{Ni}^{2+}$-NTA agarose. Separation of the complexes by SDS-PAGE analysis under nonreducing conditions (Fig. 1A) showed that all 10 single cysteine mutants were expressed and effectively dissociated into monomers with the expected size of $\sim 50 \mathrm{kDa}$. In addition, we performed BN-PAGE analysis to verify that the mutants were assembled correctly (Fig.
$1 B)$. All mutants assembled efficiently into complexes with an apparent molecular weight of $\sim 250 \mathrm{kDa}$, which corresponded to trimeric complexes as revealed by partial dissociation with SDS (note that under the native electrophoresis conditions, the mobility of the P2X complexes in relation to the soluble marker proteins does not correlate with the actual molecular weight of the P2X trimer). No intermediate complexes indicative of partially assembled or dissociated complexes were visible. Likewise, no aggregates were formed, which would have biased the subsequent experiments.

Finally, we tested the surface expression of the mutants by selective labeling of plasma membrane receptors. To this end, we treated the intact oocytes with Cy5-conjugated NHS-ester. This fluorescent compound reacts with primary amines at $\mathrm{pH}>7$ and is supposed to not enter the cell and/or to label only very inefficiently at intracellular $\mathrm{pH}$. As shown in Figure $1 C$, all mutants were labeled efficiently. Deglycosylation with EndoH confirmed that all Cy5-labeled mutants consisted of a uniform protein population carrying both EndoH-sensitive and EndoH-resistant $N$-glycans, thus excluding the presence of incompletely processed intracellular forms of the P2XR, which would have been completely deglycosylated by EndoH [(Nicke et al., 1998) results shown only for the wild-type (wt) $\mathrm{P} 2 \mathrm{X}_{1}$ subunit]. The effective expression of all mutants in the plasma membrane further shows that the introduced cysteine residues did not severely influence receptor folding, assembly, or surface transport.

\section{Identification of an intersubunit disulfide bond between K68C and F291C}

Next, we expressed all pairwise combinations of single cysteine mutants and analyzed the purified receptor complexes by nonreducing SDS-PAGE to scan for spontaneous disulfide formation between P2X subunits. Figure $2 A$ (left panel) shows the respective results for the $\mathrm{K} 68 \mathrm{C}$ mutant in combination with all other single mutants. A spontaneous formation of a band corresponding in size to a dimer was obtained with the K68C and F291C mutants. This dimer formation was even more efficient than for 


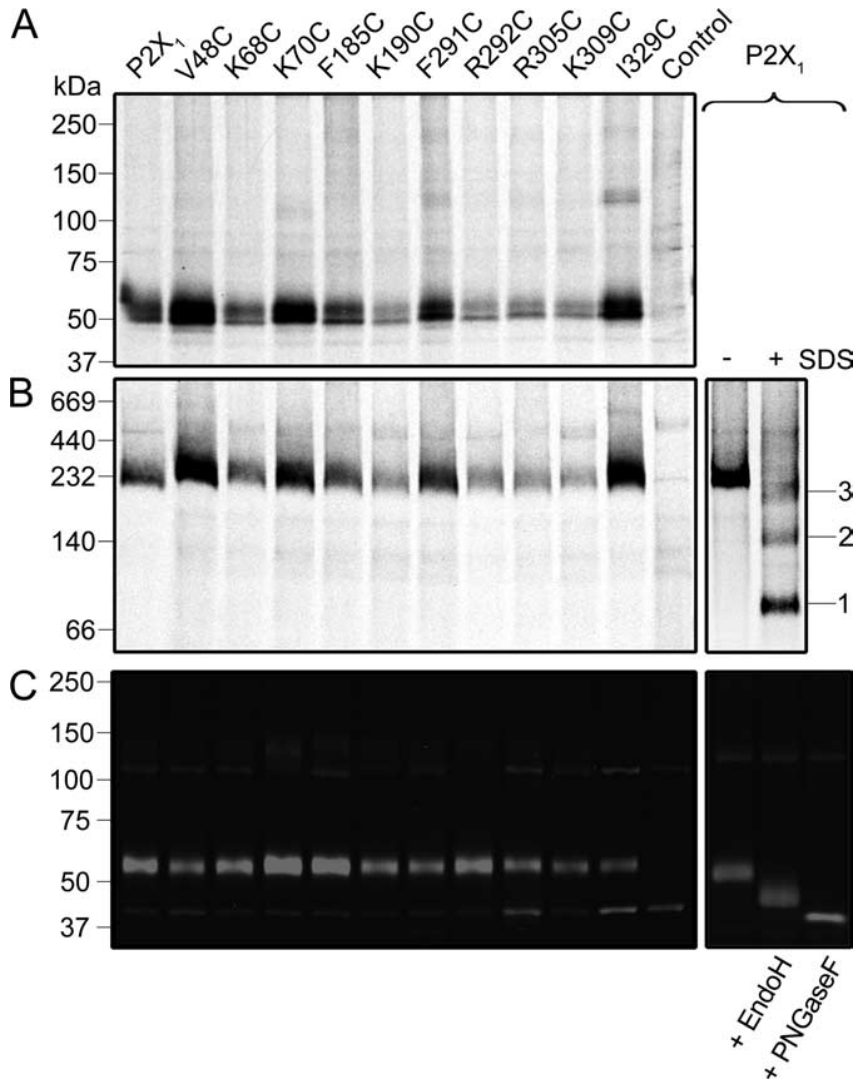

Figure 1. Validation of synthesis and correct assembly of single cysteine-substituted mutations. X. laevis oocytes injected with cRNA encoding the indicated His-tagged P2X $X_{1}$ mutants, or noninjected control oocytes, were metabolically labeled by incubation in $\left[{ }^{35} \mathrm{~S}\right]$ methionine or treated with Cy5-conjugated NHS-ester. His-tagged protein complexes were subsequently purified under nondenaturing conditions from digitonin (1\%) extracts of the 00cytes. A, Autoradiograph of metabolically labeled single cysteine mutants resolved on a nonreducing SDS-PAGE gel (8\%). $\boldsymbol{B}$, The same protein samples as in $\boldsymbol{A}$ analyzed by BN-PAGE analysis (10-13\%). Partial dissociation by SDS (right panel) confirms the trimeric structure (numbers indicate monomers, dimers, and trimers, respectively). C, Selectively labeled cell-surface protein analyzed by nonreducing SDS-PAGE (8\%). Deglycosylation analysis (right panel) shows a uniform population of complex glycosylated (EndoH resistant) protein, thus showing the specificity of the surface labeling. PNGaseF, Peptide: $N$-glycosidase F.

the V48C + I329C pair (Jiang et al., 2001, 2003), which served as a positive control.

Markedly less or no dimer formation was observed for the other combinations. Likewise, if the reverse experiment was performed with the F291C mutant in combination with all other mutants, a pronounced dimer formation was observed only in combination with the K68C mutant (Fig. $2 \mathrm{~A}$, right panel); however, the F291C mutant had an increased propensity to form dimers by itself (Fig. $2 D$ ), suggesting that this mutation promotes the formation of nonspecific disulfide bridges between subunits.

As mentioned above, intersubunit disulfide formation can occur during P2XR folding and assembly, and this may be even more pronounced if mutant subunits containing additional cysteine residues are expressed. Therefore, we could not exclude the possibility that the observed dimers represented intracellular folding intermediates or misfolded protein. To verify that the disulfide formation occurred in fully processed and mature receptors, we also analyzed the selectively labeled plasma membrane-bound P2X mutants by nonreducing SDS-PAGE. Consistent with the results described for metabolically labeled oocytes, Figure $2 B$ shows that a specific and distinctive dimer was obtained only with the coexpressed K68C and F291C subunits.
As shown in Figure 3C, the dimers could be completely dissociated into monomers if SDS-PAGE was performed under reducing conditions (addition of $20 \mathrm{~mm}$ DTT to the protein sample). This confirms that dimerization was caused by the formation of disulfide bridges. All other pairwise combinations of single cysteine mutants did not result in the formation of substantial amounts of dimers (results not shown). To exclude the possibility that the cross-link resulted from aggregation between receptor complexes, the coexpressed single mutants were also analyzed by nondissociating BN-PAGE. Figure $2 C$ shows that all combinations formed trimeric complexes, and no aggregation between receptor complexes occurred.

The absence of higher adducts like trimers or tetramers is in agreement with a specific cross-link between neighboring residues within a trimeric complex (Fig. $2 E$ ). Together, these experiments show a specific intersubunit disulfide formation between the $\mathrm{K} 68 \mathrm{C}$ and $\mathrm{F} 291 \mathrm{C}$ mutants, indicating a close proximity of these residues.

\section{K68C/F291C double-cysteine mutants form disulfide-linked trimers}

If the $\mathrm{K} 68 \mathrm{C}$ and $\mathrm{F} 291 \mathrm{C}$ mutants were coexpressed, $\sim 40-50 \%$ of the total protein was found to be present as a dimer (Fig. 2D). This is in good agreement with the calculated probability of K68C + F291C-heteromer formation (50\%) if equal amounts of both mutants were expressed (Fig. $2 E$ ). An $\sim 10 \%$ reduced dimer formation was observed in the plasma membrane, suggesting a less efficient cross-linking in the mature receptor. It has to be considered, however, that the maximum value of $50 \%$ dimer formation in the plasma membrane can be obtained only if (1) both mutants are expressed with a similar efficiency and time course and (2) assembled in a purely stochastic manner, and (3) homomeric or heteromeric complexes of both mutants are transported to the plasma membrane with equal efficiency. Quantification and comparison of the metabolically labeled total amount of singly expressed K68C and F291C mutants and their selectively labeled plasma membrane forms revealed that both mutants are expressed in similar amounts but that the F291C mutant is transported approximately fivefold less efficiently to the plasma membrane (results not shown). Thus, it is likely that the apparently inefficient cross-linking in the plasma membrane reflects the favored plasma membrane transport of the homomeric $\mathrm{K} 68 \mathrm{C}$ mutant.

The amount of metabolically labeled dimer was not markedly increased if the purified protein was treated with oxidizing agents such as hydrogen peroxide, $\mathrm{Cu}^{2+}$ phenantroline, or iodine (results not shown). Reduction of the $\mathrm{Ni}^{2+}$-bound protein sample with $20 \mathrm{~mm} \beta$-mercaptoethanol and subsequent oxidation of the eluted sample by the addition of $2 \mathrm{~mm}$ hydrogen peroxide produced a similar amount of $\sim 40 \%$ cross-linked product (Fig. $3 E$; supplemental Fig. $1 \mathrm{~A}$, available at www.jneurosci.org as supplemental material), thus further showing the high efficiency of spontaneous disulfide formation.

Assuming a circular and symmetrical $\mathrm{P}_{2} \mathrm{X}_{1} \mathrm{R}$ complex with three equivalent ATP-binding sites, it should be possible to crosslink all three subunits if mutations K68C and F291C were both introduced into a single subunit (Fig. $3 B$ ). As expected, the respective double mutants largely cross-linked into disulfidelinked complexes of $\sim 150 \mathrm{kDa}$, the predicted size of a $\mathrm{P} 2 \mathrm{X}_{1}$ trimer (Fig. 3A); however, an additional band of $\sim 200 \mathrm{kDa}$, corresponding in size to a tetramer, was also observed (indicated by star symbol). This could indicate that the cross-linked subunits derived from unspecifically aggregated complexes rather 
than being formed within correctly assembled trimers. This possibility was excluded by BN-PAGE analysis (Fig. 3D), which showed that the double mutant, like the respective single mutants, migrated as a distinct trimer. Therefore we conclude that the high molecular weight complex observed by nonreducing SDS-PAGE was artificially formed by disulfide linkage of two incompletely cross-linked receptor complexes after the addition of SDS. Alternatively, the 150 and $200 \mathrm{kDa}$ bands could represent completely (circularly) and incompletely (linearly) cross-linked forms of trimers (Fig. 3B), which are likely to show differences in their mobility on a gradient gel because of their different Stokes dimensions in the extended or globular conformations. In support of our findings, a very similar pattern of two bands close to the size expected for a trimer has been found for $\mathrm{P}_{2} \mathrm{X}_{2} \mathrm{Rs}$, in which an intersubunit cross-link was identified between two cysteine residues substituting for a pair of histidine residues that contributes to the formation of the $\mathrm{Zn}^{2+}$ binding site (Nagaya et al., 2005).

A weak band corresponding to a dimer (Fig. $3 A, F$ ) suggests that formation of the first disulfide bridge in the double mutant might hold the protein in a more rigid conformation, which in turn may impede formation of the second and third disulfide bridges; however, the fact that hardly any monomer is seen supports the idea that at least the first disulfide bridge is formed with a high efficiency.

The formation of an intersubunit disulfide bond between $\mathrm{K} 68 \mathrm{C}$ - and F291C-substituted residues is prevented in the presence of ATP

As described above, residues $\mathrm{K} 68$ and F291 are presumed to contribute to ATP binding. Our biochemical data show that these two residues, if substituted by cysteines, can form a specific disulfide crosslink across a subunit interface, suggesting that the ATP-binding site is formed between neighboring subunits. If so, binding of ATP should influence or prevent disulfide bond formation. To test this hypothesis, we expressed the K68C and F291C single-mutant pair and the respective double-mutant subunits and treated the respective receptor complexes during the purification process, while they were still bound to the $\mathrm{Ni}^{2+}$-NTA agarose beads, with $20 \mathrm{~mm} \beta$-mercaptoethanol. Elution was then performed under nondenaturing conditions in the presence of $10 \mathrm{mM}$ ADP or ATP (Fig. $3 E, F$ ) or in the presence and absence of $100 \mathrm{~mm}$ ATP (supplemental
A

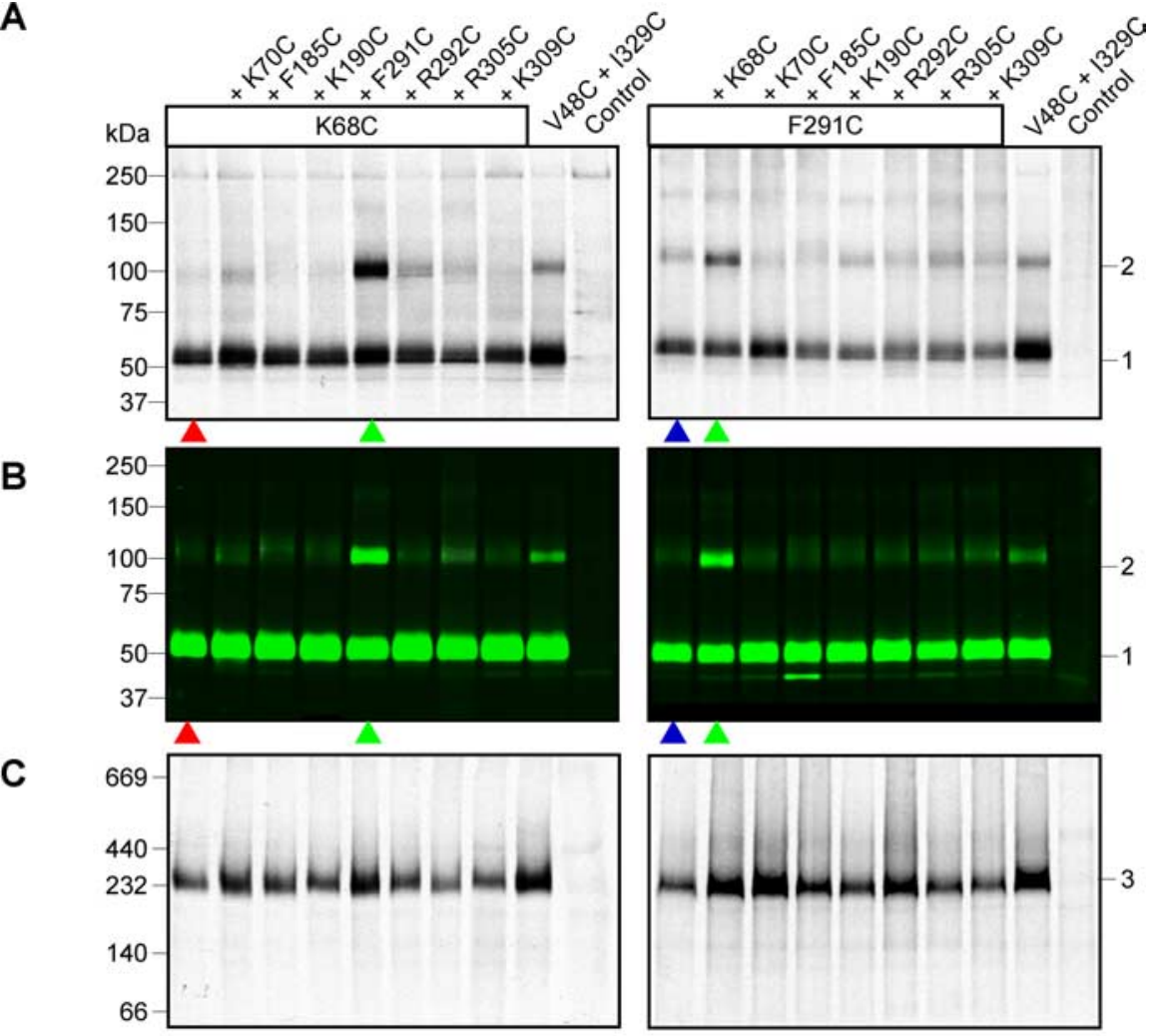

D
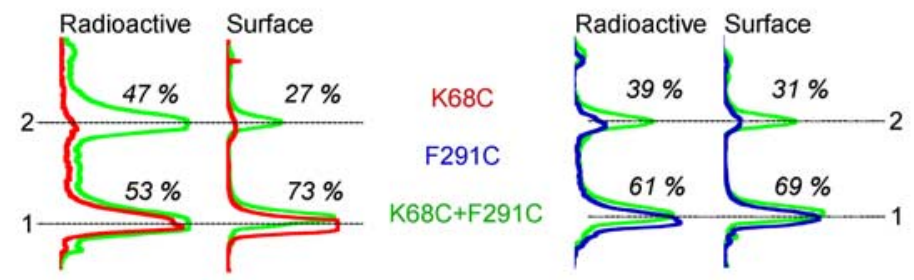

E

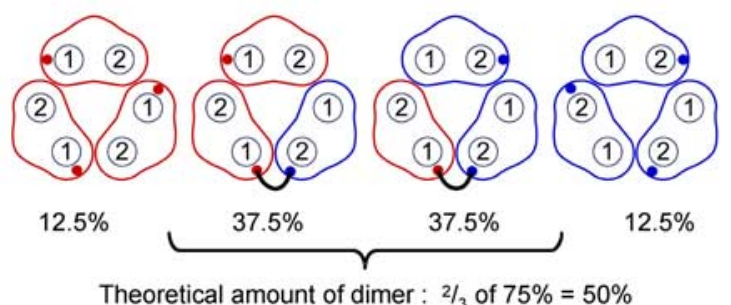

Figure 2. Screening for specific intersubunit disulfide bond formation by pairwise coexpression of single cysteine mutants. The indicated mutant His-P2X 1 subunits were coexpressed in Xenopus 0ocytes. P2X complexes were purified via Ni ${ }^{2+}$-NTA agarose and analyzed for specific dimer formation (numbers indicate the oligomeric state of the corresponding protein bands). Controls are from noninjected oocytes. $\boldsymbol{A}, \boldsymbol{B}$, Nonreducing SDS-PAGE analysis of metabolically labeled total protein $(\boldsymbol{A})$ and $(y 5$-labeled plasma membrane protein $(\boldsymbol{B})$ complexes formed after coexpression of the K68C (left panel) and F291C (right panel) mutants in combination with all other mutants. The V48C + I329C pair (Jiang et al., 2001, 2003) served as a positive control. The singly expressed K68C and F291C mutants served as negative controls to estimate the background of unspecific disulfide bond formation. Note the specific dimer formation for coexpressed K68C and F291C mutants. C, BN-PAGE analysis of metabolically labeled complexes formed after coexpression of single cysteine mutants. $\boldsymbol{D}$, Relative amounts of dimers and monomers analyzed by phosphor imaging or fluorescence scanning of the gels shown in $\boldsymbol{A}$ and $\boldsymbol{B}$. Intensity profiles of lanes with single and coexpressed K68C and F291C mutants from the same gels are compared. Like-colored triangles in $\boldsymbol{A}$ and $\boldsymbol{B}$ indicate the lanes of singly expressed K68C (red) and K291C (blue) mutants and coexpressed K68C + K291C mutants (green) that were analyzed. $\boldsymbol{E}$, Calculated probabilities for the formation of homomeric and heteromeric subunit combinations and expected ratio of dimeric protein, if both subunits are expressed in equal amounts. Note that the total amount of receptors containing cross-linked subunits (75\%) has to be multiplied by two-thirds because only two of three subunits migrate as a dimer after dissociation by SDS. Subunits are schematically represented with two transmembrane domains with the $\mathrm{K} 68 \mathrm{C}$ and $\mathrm{F} 291 \mathrm{C}$ mutations (solid circles) located on the same side as the first and second transmembrane domain, respectively. A solid line between adjacent mutations represents a disulfide bond formation. 
A
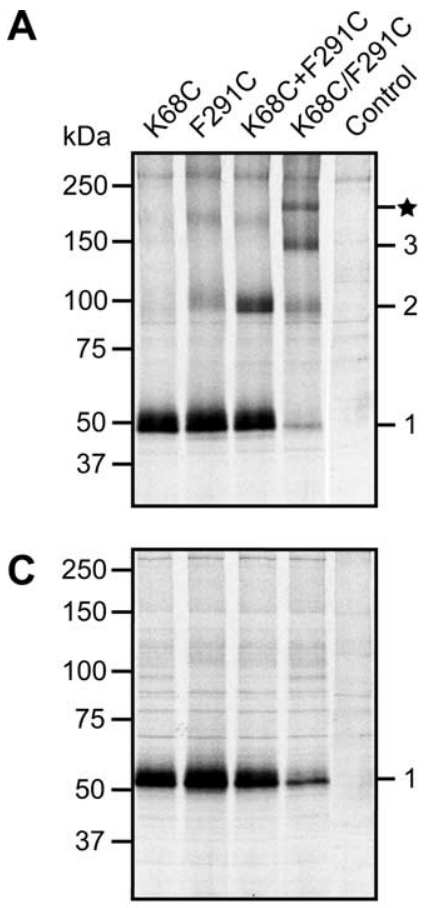

E

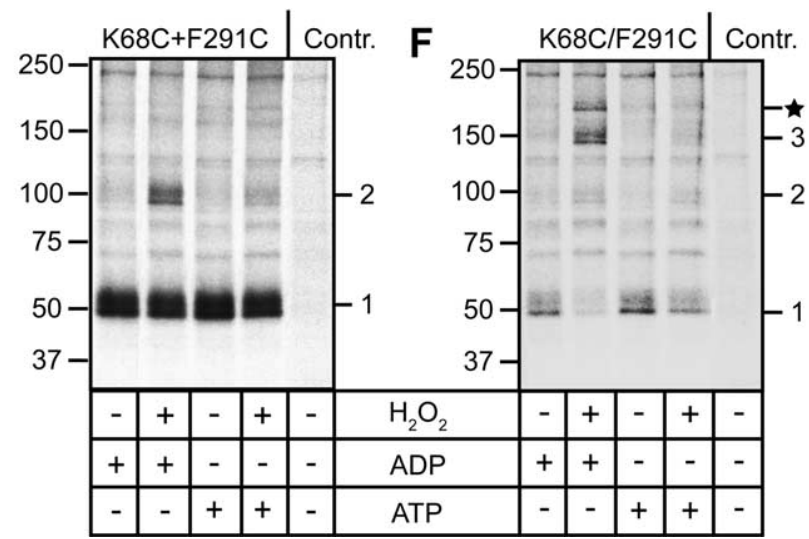

Figure 3. Biochemical analysis of the $\mathrm{K} 68 \mathrm{C} / \mathrm{F} 291 \mathrm{C}$ double mutant and inhibition of disulfide bond formation by ATP. Xenopus oocytes injected with cRNAs encoding the indicated mutations of His-tagged P2X $\mathrm{X}_{1}$ subunits and noninjected control oocytes were metabolically labeled by overnight incubation in $\left[{ }^{35} S\right.$ ] methionine. $A, C, D$, His-tagged protein complexes were purified via $\mathrm{Ni}^{2+}$-NTA agarose from digitonin (1\%) extracts and analyzed by nonreducing SDS-PAGE $(\boldsymbol{A})$, reducing SDS-PAGE $(\boldsymbol{C})$, and BN-PAGE $(\boldsymbol{D})$. B, Schematic illustration of the expected globular ring and linear conformations of the cross-linked $K 68 \mathrm{C} / F 291 \mathrm{C}$ double mutant, which could account for the additional protein band in $\boldsymbol{A}$ (indicated by star symbol). $\boldsymbol{E}, \boldsymbol{F}$, Comparison of disulfide bond formation in receptor complexes that were subjected to reducing treatment while bound to the $\mathrm{Ni}^{2+}$-NTA agarose beads, subsequently eluted in the presence of $10 \mathrm{~mm} \mathrm{ATP}$ or ADP (control) and treated with or without $2 \mathrm{~mm} \mathrm{H}_{2} \mathrm{O}_{2}$ before separation on a nonreducing SDS-PAGE gel. Results are shown for coexpressed K68C and F291C single mutants $(\boldsymbol{E})$ and the respective double mutant $(\boldsymbol{F})$. See also supplemental Figure 1, available at www.jneurosci.org as supplemental material. Contr., Control.

Fig. $1 A$, available at www.jneurosci.org as supplemental material). Previous studies (Nicke et al., 1998) and control experiments by BN-PAGE (results not shown) had shown that this reducing treatment does not affect the trimeric quaternary structure of the P2X complexes, suggesting that the native intrasubunit disulfide bridges of the $\mathrm{P} 2 \mathrm{X}_{1} \mathrm{R}$ are not accessible under nondissociating conditions. SDS-PAGE analysis under nonreducing conditions confirmed that this treatment efficiently reduced the intersubunit disulfide bridges (Fig. $3 E$, F; supplemental Fig. 1, available at www.jneurosci.org as supplemental material).
The addition of $2 \mathrm{mM} \mathrm{H}_{2} \mathrm{O}_{2}$ resulted in an efficient reformation of the cross-link, which was clearly inhibited when the protein sample was eluted in the presence of ATP. In contrast, ADP, which is not an agonist at P2XRs (Mahaut-Smith et al., 2000), did not inhibit disulfide bond formation (Fig. $3 E, F$ ). The specificity of the ATP effect is further shown by the fact that disulfide bond formation between V48C- and I329C-substituted residues was not inhibited by ATP (supplemental Fig. 1C, available at www.jneurosci.org as supplemental material). This finding is in accord with the idea that binding of ATP prevents the close interaction of the $\mathrm{K} 68 \mathrm{C}$ and $\mathrm{F} 291 \mathrm{C}$ residues either by directly interacting with one or both of these residues or by locking the receptor in a conformation in which the close approximation of these residues is constrained.

\section{Functional analysis of the single cysteine substitutions}

All P2X $\mathrm{X}_{1}$ mutants were tested for functionality in two-electrode voltage-clamp experiments and displayed currents in the microampere range when activated by 3 mM ATP (data not shown). Because the cysteine substitutions were introduced at positions presumed to contribute to ATP binding, the dose-response curves for ATP were shifted to the right ( 7 - to $>700$-fold) at these mutants (data not shown). Together with the fast desensitizing properties of the $\mathrm{P} 2 \mathrm{X}_{1} \mathrm{R}$, this complicated their functional characterization. Therefore, we made use of a $\mathrm{P} 2 \mathrm{X}_{2-1}$ chimera, in which the $\mathrm{N}$ terminus and the first transmembrane domain of the $\mathrm{P} 2 \mathrm{X}_{1} \mathrm{R}$ subunit were replaced by the corresponding $\mathrm{P} 2 \mathrm{X}_{2}$ sequence (Fig. $4 \mathrm{~A}$ ). This chimera shares the nondesensitizing properties of the P2X $\mathrm{X}_{2} \mathrm{R}$ (Werner et al., 1996) and, in contrast to the wt $\mathrm{P} 2 \mathrm{X}_{1} \mathrm{R}$, exhibits a nanomolar ATP sensitivity. Two explanations have been suggested for this high ATP sensitivity: (1) both wt and chimeric receptor binding sites have the same high ATP affinity that, in the wt $\mathrm{P} 2 \mathrm{X}_{1} \mathrm{R}$, is masked by desensitization (Rettinger and Schmalzing, 2003, 2004), or (2) a gating-associated lock of agonist results in the increased ATP sensitivity (Zemkova et al., 2004). Functional studies have revealed virtually identical potencies of the competitive antagonist NF279 at the $\mathrm{P}_{2} \mathrm{X}_{1} \mathrm{R}$ and the chimera, thus confirming that the latter provides a valid model for the ATP-binding site of the $\mathrm{P}_{2} \mathrm{X}_{1} \mathrm{R}$ (Rettinger and Schmalzing, 2004). Biochemical analysis of the chimera further showed that it displayed the same assembly and glycosylation patterns as the wt $\mathrm{P} 2 \mathrm{X}_{1}$ protein (supplemental Fig. 2, available at www.jneurosci.org as supplemental material). Its high sensitivity to ATP $\left(\mathrm{EC}_{50} \sim 3 \mathrm{nM}\right)$ allows functional analysis of mutations that reduce ATP potency $\sim 100,000$-fold and enabled the construction of dose-response curves for all cysteine mutants except the R305C mutant (Fig. 4B,E, Table 2). The R305C mutant was functional but displayed continuously increasing inward currents after ATP application, which hampered the construction of reliable doseresponse curves. In agreement with the respective alanine substitutions at the $\mathrm{P} 2 \mathrm{X}_{1} \mathrm{R}$ (Table 1), among the greatest shifts in $\mathrm{EC}_{50}$ values for ATP were caused by substitution of the K68 and K309 residues ( $\sim 60,000$-fold and 7000 -fold respectively). In addition, the K70C mutation caused a strong reduction in ATP sensitivity (8500-fold shift). All other cysteine mutations in the chimera caused changes in ATP potency that were in the same range or up to 50 -fold larger than those observed for the corresponding alanine substitutions in the $\mathrm{P} 2 \mathrm{X}_{1} \mathrm{R}$. The observed differences in absolute changes might reflect the different substitutions (alanine versus cysteine) and/or the desensitizing properties of the $\mathrm{P} 2 \mathrm{X}_{1}$ mutants.

In conclusion, all of our cysteine mutants were functionally expressed and could be characterized with the $\mathrm{P} 2 \mathrm{X}_{2-1}$ chimera as 
a model. They displayed nondesensitizing currents in the microampere range (Table 2 ), further showing that the cysteine substitutions did not grossly disturb correct receptor folding and function but markedly influenced their responsiveness to ATP. More importantly, the nondesensitizing properties of the chimera allowed the investigation of the time course of disulfide bond formation (Fig. $4 C, F, G$ ).

\section{The $\mathrm{K68C} / \mathrm{F} 291 \mathrm{C}$ double mutant is} rendered functional after treatment with DTT

To directly investigate the consequences of the K68C/F291C disulfide formation for receptor function, we next analyzed the respective double mutant in the $\mathrm{P}_{2 \mathrm{X}_{2-1}}$ chimera. Application of $300 \mu \mathrm{M}$ ATP revealed only minimal currents of 10-20 nA, suggesting that the intersubunit disulfide bond might block the access of ATP to its binding site and/or inhibit conformational rearrangements required for channel opening. To test this assumption, we superfused the oocyte with $10 \mathrm{~mm}$ DTT. This reducing treatment does not alter the function of the nonmutated chimera (results not shown). In the $\mathrm{K} 68 \mathrm{C} /$ F291C chimera, DTT increased ATPinduced currents by $63 \pm 11$-fold as compared with the initial responses before DTT application (Fig. 4C,D). This indicates that $<2 \%(1 / 60)$ of the doublemutant receptors did not contain intersubunit disulfide bridges and further supports the high efficiency of spontaneous disulfide bond formation between K68C- and F291C-substituted residues.

Similar results were obtained with the reducing agent Tris(2-carboxyethyl) phosphine hydrochloride (results not shown). The DTT-induced current increase saturated within $\sim 15 \mathrm{~min}$, and currents remained stable or showed only a small decrease $(<10 \%)$ after repeated application of $300 \mu \mathrm{M}$ ATP. As expected, extracellular application of $2 \mathrm{mM} \mathrm{H}_{2} \mathrm{O}_{2}$ reversed the current increase, thus showing that disulfide bond formation occurs in functional receptors and does not result from artifactual misfolding after insertion into the plasma membrane (Fig. 4C).

As a control, the respective single mutants K68C and F291C were also tested for their sensitivity to DTT (Fig. 4D). The F291C mutant showed large inward currents when activated by $300 \mu \mathrm{M}$ ATP that were not changed after extracellular application of $10 \mathrm{~mm}$ DTT. In contrast, the K68C mutant displayed a rapid approximately threefold current increase immediately after application of extracellular
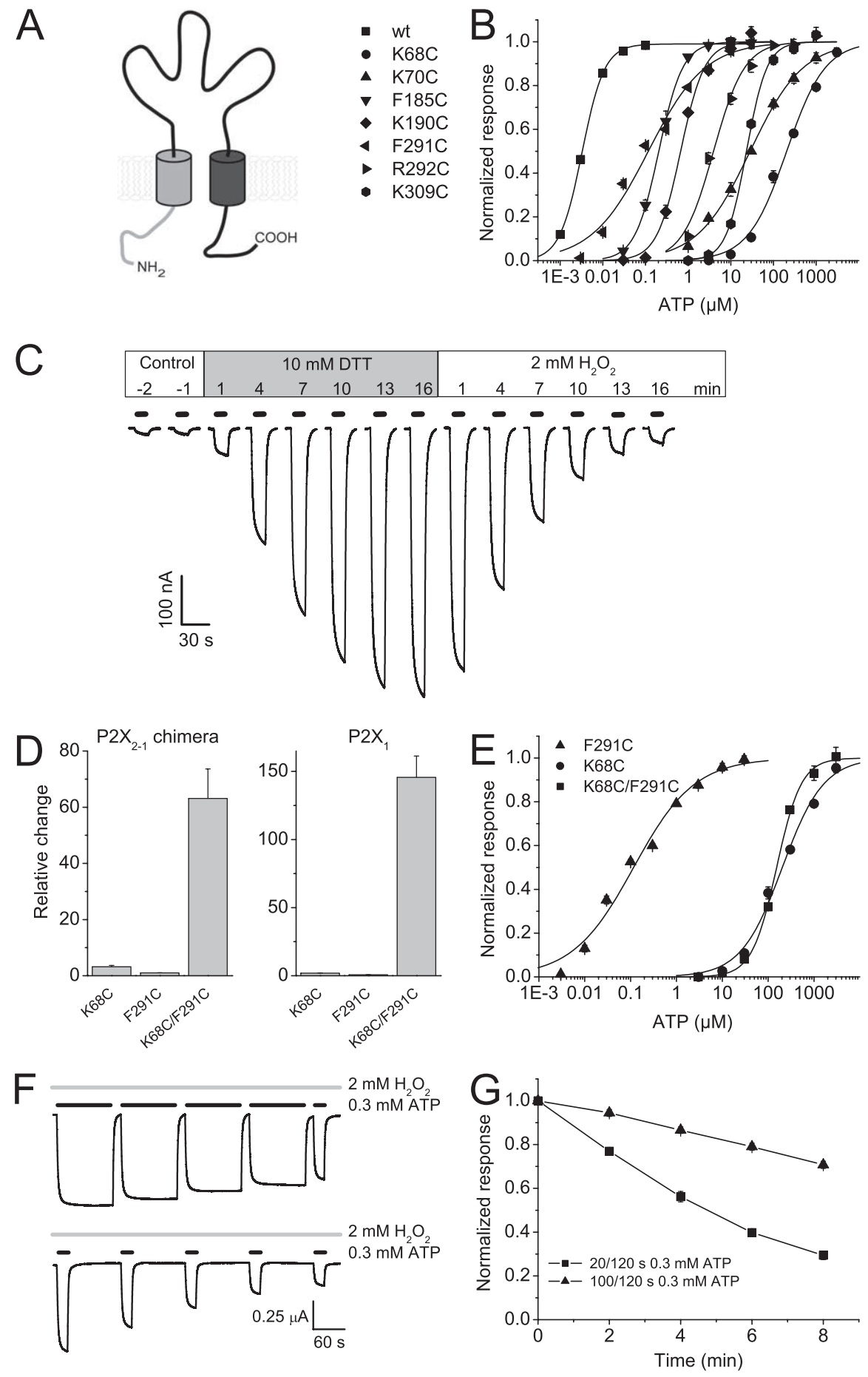

Figure 4. Two-electrode voltage-clamp analysis of the single cysteine mutations and the K68C/F291C double mutation in a $\mathrm{P} 2 \mathrm{X}_{2-1}$ chimera. $\boldsymbol{A}$, Schematic representation of the $\mathrm{P} 2 \mathrm{X}_{2-1}$ chimera in which the $\mathrm{N}$ terminus and first transmembrane domain of the $P 2 X_{1} R$ (black line) were replaced by the corresponding $P 2 X_{2} R$ sequence (gray line). $B$, Dose-response curves for ATP of wt and single cysteine mutant receptors. Lines represent nonlinear curve-fits of the Hill equation to the data. Data were normalized to the $I_{\max }$ value derived from the curve fit for better comparability (for $\mathrm{EC}_{50}$ values and Hill coefficients, see Table 2). C, Original current recording from an 0ocyte expressing the $K 68 C / F 291$ C double-mutant receptor. After obtaining stable responses after application of $300 \mu \mathrm{m} \mathrm{ATP}$, the oocyte was superfused with $10 \mathrm{~mm}$ DTT; this caused a $\sim 50$-fold increase in receptor current. Application of 2 $\mathrm{mm} \mathrm{H}_{2} \mathrm{O}_{2}$ reversed the DTT effect. The holding potential was $-40 \mathrm{mV}$. D, Effect of DTT on cysteine-mutated P2X $\mathrm{X}_{2-1}$ chimeras and P2X $X_{1}$ Rs. Oocytes were preincubated in $10 \mathrm{~mm}$ DTT before current measurements in response to $300 \mu \mathrm{m}$ ATP. E, ATP dose-response curves for the K68C/F291C double mutant after preincubation in $10 \mathrm{~mm}$ DTT for at least $15 \mathrm{~min}$. For comparison, the dose-response curves of the respective single-mutant receptors (as in $\boldsymbol{B}$ ) are also shown (for $\mathrm{EC}_{50}$ values and Hill coefficients, see Table 2). $\boldsymbol{F}, \boldsymbol{G}$, Disulfide bond formation between $\mathrm{K} 68 \mathrm{C}$ and F291C in the double mutant is inhibited in the presence of ATP. After 15 min preincubation in $10 \mathrm{~mm}$ DTT, current responses to $0.3 \mathrm{~mm} \mathrm{ATP}$ were determined in the continuous presence of $2 \mathrm{~mm} \mathrm{H}_{2} \mathrm{O}_{2}$. ATP was applied in 2 min intervals, either for $20 \mathrm{~s}$ (squares) or for $100 \mathrm{~s}$ (triangles). Error bars represent SEM; $n=5$ for each protocol. Responses from control oocytes (recorded in the absence of $\mathrm{H}_{2} \mathrm{O}_{2}$ ) declined $<10 \%$ in both protocols (results not shown). 
Table 2. Summary of $\mathrm{EC}_{50}$ values of ATP at single cysteine substituted mutants in the $\mathrm{P}_{2 \mathrm{X}_{2-1}}$ chimera

\begin{tabular}{lccrc}
\hline P2X $X_{2-1}$ chimera & $\mathrm{EC}_{50}(\mu \mathrm{M})$ & $n_{\mathrm{h}}$ & $n$ & $I_{\max }(\mu \mathrm{A})$ \\
\hline Non-mutated & $0.0033 \pm 0.0001$ & $1.66 \pm 0.04$ & $6-8$ & $3.2 \pm 0.3$ \\
K68C & $204 \pm 44$ & $0.96 \pm 0.14$ & $9-12$ & $4.9 \pm 0.7$ \\
K70C & $28 \pm 4$ & $0.7 \pm 0.04$ & $11-12$ & $13.3 \pm 1.7$ \\
F185C & $0.21 \pm 0.004$ & $1.55 \pm 0.04$ & 18 & $4.9 \pm 0.7$ \\
K190C & $0.67 \pm 0.07$ & $1.6 \pm 0.2$ & 20 & $2.9 \pm 0.5$ \\
F291C & $0.12 \pm 0.03$ & $0.7 \pm 0.1$ & $8-16$ & $5.9 \pm 0.6$ \\
R292C & $3.9 \pm 0.5$ & $1.2 \pm 0.2$ & $13-14$ & $7.1 \pm 0.9$ \\
R305C & n.d. & n.d. & & \\
K309C & $23 \pm 1$ & $1.8 \pm 0.2$ & $8-18$ & $2.3 \pm 0.3$ \\
K68C/F291C (reduced) & $153 \pm 8$ & $1.6 \pm 0.1$ & $6-11$ & $0.67 \pm 0.07$ \\
K68C/F291C (oxidized) & n.d. & n.d. & $6-11$ & $0.012 \pm 0.001$ \\
K68C/K309C & n.f. & n.f. & & n.f. \\
K68C/K309C + wt & $1.7 \pm 0.1$ & $1.5 \pm 0.1$ & 10 & $3.5 \pm 0.5$ \\
\hline
\end{tabular}

$n_{\mathrm{h}}$ is the Hill coefficient, and $n$ represents the number of oocytes used per data point. The R305C mutant was functional but showed continuously increasing currents, thus complicating the determination of reliable $\mathrm{EC}_{50}$ values. The $\mathrm{K} 68 \mathrm{C} / \mathrm{K} 309 \mathrm{C}$ mutant was not functional at concentrations up to $1 \mathrm{~mm}$ ATP. Note that the ATP sensitivity of the $P 2 X_{2-1}$ chimera is $\sim 300$ times higher than the apparent sensitivity of the P2X receptor (Rettinger and Schmalzing, 2004). n.d. and n.f. indicate mutations that were not investigated or not functional, respectively.

DTT, which in some cells reversed back to control currents after 20 min in the continuous presence of DTT. Although we have no explanation for this behavior, the clearly different time course and partial reversibility of this effect, as well as the small current increase as compared with the double mutant, suggest that this potentiation does not reflect disulfide bond reduction. A qualitatively identical effect of DTT on receptor function was found for the $\mathrm{K} 68 \mathrm{C}$ and $\mathrm{F} 291 \mathrm{C}$ single-mutant and double-mutant P2X $\mathrm{X}_{1} \mathrm{R}$ subunits (Fig. 4D). This further shows that the chimera is a valid model for studying the ATP-binding mechanism of the $\mathrm{P} 2 \mathrm{X}_{1} \mathrm{R}$.

Dose-response analysis at the reduced double-mutant chimera revealed an $\mathrm{EC}_{50}$ value of $153 \pm 8 \mu \mathrm{M}$ for ATP, a value not significantly different from that determined for the K68C single mutant (Fig. $4 E$, Table 2). It seems plausible that the $\mathrm{K} 68 \mathrm{C} /$ F291C double mutant would have a lower ATP sensitivity than the $\mathrm{K} 68 \mathrm{C}$ single mutant if both residues were directly involved in ATP binding; however, under the assumption that the F291 residue is only stabilizing the dominant interaction of ATP with the nearby positive charge in position 68 , its contribution to binding would become insignificant if the interaction with K68 is prevented. Another possible explanation for the apparently missing effect of the F291C exchange would be that in the absence of a positive charge in position 68, ATP acquires an orientation in which the interaction with F291 is prevented. Alternatively, only K68 could be directly involved in binding, whereas F291 is required to stabilize the architecture of the binding pocket or transduce the subsequent gating movement.

To directly show the interaction between ATP binding and disulfide bond formation at the functional double-mutant chimera, we investigated the influence of ATP on the reoxidation by $\mathrm{H}_{2} \mathrm{O}_{2}$. To this end, we compared the efficiency of reoxidation after short (20 s) and long-lasting (100 s) applications of $300 \mu \mathrm{M}$ ATP. In agreement with our biochemical studies at the $\mathrm{P} 2 \mathrm{X}_{1} \mathrm{R}$, the disulfide bond formation between K68C and F291C in the double-mutant chimera was significantly prevented in the continued presence of ATP (Fig. $4 F, G$ ). This finding confirms the mutual occlusion of ATP binding and disulfide bond formation and further shows the particular suitability of the nondesensitizing chimera as a $\mathrm{P} 2 \mathrm{X}_{1}$ model in these kinds of functional studies.

A similar effect of DTT and $\mathrm{H}_{2} \mathrm{O}_{2}$ on channel function as described above has also been reported by Nagaya et al. (2005) for a $\mathrm{P}_{2} \mathrm{X}_{2} \mathrm{H} 120 \mathrm{C} / \mathrm{H} 213 \mathrm{C}$ double mutant and can be expected for any disulfide cross-link that inhibits movements involved in channel opening, independently of whether the cross-linked res- idues are involved in binding or gating or just need to move freely during opening. The mutations investigated by Nagaya et al., (2005), however, did not directly influence ATP potency but instead were convincingly shown to be involved in the formation of an intersubunit $\mathrm{Zn}^{2+}$-binding site; hence they allosterically modulate ATP potency.

\section{The function of the $\mathrm{K} 68 \mathrm{C} / \mathrm{K} 309 \mathrm{C}$ double-cysteine mutant can be rescued by coexpression of the wt-chimera}

The strongest effects on ATP-activated currents have been described for alanine substitutions of the $\mathrm{K} 68$ or $\mathrm{K} 309$ residues in $\mathrm{P} 2 \mathrm{X}_{1}$ and the corresponding residues in $\mathrm{P}_{2} \mathrm{X}_{2}$ (Ennion et al., 2000; Jiang et al., 2000) (Table 1) and were also found here with the respective cysteine substitutions in the $\mathrm{P} 2 \mathrm{X}_{2-1}$ chimera. Because K68 and K309 are located at opposite ends of the extracellular region, close to the transmembrane domains, and in equal distance ( 20 amino acid residues) from the V48 and I329 residues (Jiang et al. 2003) (compare Fig. 6), we considered it likely that they are also contributed by neighboring subunits. Biochemical experiments with and without cysteine-specific cross-linkers, however, did not provide support for a close proximity of these residues from neighboring subunits (results not shown). Therefore, we performed functional studies with the respective double mutant in the $\mathrm{P}_{2} \mathrm{X}_{2-1}$ chimera. If expressed alone, no currents could be evoked by concentrations up to $1 \mathrm{~mm}$ ATP, neither before nor after treatment with DTT. Thus, combined substitution of these two critical residues completely disabled ATP binding or subsequent channel opening. If, however, the $\mathrm{K} 68 \mathrm{C} / \mathrm{K} 309 \mathrm{C}$ double mutant was coexpressed with the nonmutated chimeric subunit (cRNA ratio 1:1), a biphasic ATPactivated current was observed (Fig. 5A), indicating that the double mutant could be functionally rescued by heteromerization with the nonmutated subunit (Fig. 5C). In addition to the homomeric wt-chimera, which showed maximal currents at $\geq 100 \mathrm{nM}$ ATP and could be clearly distinguished by a very slow time course of channel closure (deactivation time constant $\sim 60 \mathrm{~s}$ ) after removal of ATP (Fig. 5A, inset) (see also Rettinger and Schmalzing, 2004), a presumptive heteromeric receptor was observed that was maximally activated by $\geq 30 \mu \mathrm{M} \operatorname{ATP}\left(\mathrm{EC}_{50}=1.7 \pm 0.1 \mu \mathrm{M}\right.$; Hill coefficient $n_{\mathrm{h}}=1.5 \pm 0.1$ ) (Fig. $5 B$ ) and that closed immediately after agonist washout. Under the assumption that three ATP molecules are required to open the channel, these findings indicate that K68 and K309 from different subunits function together to elicit ATP-induced channel opening and that at least one of these two residues per interface is required for that function. As depicted in Figure 5C, heteromers of two nonmutated subunits with one double-mutant subunit would still form channels with three functional interfaces, whereas only two functional interfaces can be expected in heteromers containing two doublemutant subunits.

\section{Discussion}

In this study, we generated eight $\mathrm{P}_{2} \mathrm{X}_{1}$ subunits in which single residues presumed to contribute to ATP binding were replaced by cysteine residues. By pairwise coexpression of all 28 combinations of these singly mutated subunits, we could identify two residues, K68C and F291C, which efficiently form a spontaneous 
and specific disulfide cross-link between two P2X subunits. By selective surface labeling and BN-PAGE analysis, we confirmed that all single and pairwise expressed mutants assembled into trimeric complexes and were correctly inserted into the plasma membrane, thus excluding the possibility that disulfide bond formation occurred artifactually as a result of protein misfolding and aggregation. In agreement with a close proximity of the identified residues within a trimeric complex, the K68C/F291C doublemutant receptor was readily cross-linked into a trimer. Both dimer formation of coexpressed single-mutant subunits and trimer formation of the double-mutant were specifically prevented in the presence of ATP, thus providing additional support for the involvement of these residues in ATP binding. The functional consequences of $\mathrm{K} 68 \mathrm{C} / \mathrm{F} 291 \mathrm{C}$ disulfide bond formation were further studied in a nondesensitizing $\mathrm{P} 2 \mathrm{X}_{2-1}$ chimera, which has previously been shown to mimic the ligand-binding properties of the $\mathrm{P} 2 \mathrm{X}_{1} \mathrm{R}$ (Rettinger and Schmalzing, 2004). Twoelectrode voltage-clamp analysis of the K68C/F291C double mutant revealed that the disulfide cross-link could be reversibly reduced and reoxidized, thereby rendering the receptor functional and nonfunctional, respectively. Hence, the two cysteine substitutions do not prevent ATP binding and receptor activation unless they are forming a disulfide bond. Once the cysteine cross-link is formed, however, ATP-binding and channel opening are prevented or at least strongly reduced. The simplest interpretation of these findings is that the ATP binding site is formed between two neighboring subunits (Fig. 6), similar to the agonist-binding site in the nAChR family members. This interpretation is also consistent with our observation that the K68C/K309C double mutant, which contains the two mutations resulting in the largest effects on ATP sensitivity, is nonfunctional if expressed alone but can be functionally rescued if coexpressed with nonmutated subunits (Fig. 5).

\section{ATP binding or gating: functional considerations}

Our conclusions stated above critically depend on the assumption that the residues mutated in our study contribute directly to ATP binding. Most published mutagenesis studies aiming to determine the amino acids involved in ATP binding or pore opening rely primarily on the interpretation of altered agonist potencies; however, changes in ATP potency at mutant receptors can reflect a reduced ATP binding affinity or an altered efficacy of the transition from the closed to the open channel conformation, or both (Colquhoun, 1998). An unequivocal answer to the question whether changes in apparent agonist affinity originate from altered binding or gating properties comes from the statistical anal- ysis of open and closed time distributions derived from singlechannel recordings of the respective mutant. So far, no singlechannel study has been performed for any of the supposed binding site mutants of P2XRs. Thus we cannot exclude the possibility that residues identified in this study are involved in the gating rather than the binding process; however, a direct interaction of the region around K68 with ATP is supported by data obtained in a substituted cysteine accessibility study of the region from D57-K71 of the $\mathrm{P}_{2} \mathrm{X}_{2} \mathrm{R}$ (corresponding to T57-K70 in $\mathrm{P} 2 \mathrm{X}_{1}$ ) (Jiang et al., 2000). In this study, ATP dose-response relationships at the $\mathrm{S} 65 \mathrm{C}$ and $\mathrm{I} 67 \mathrm{C}$ mutants (corresponding to S64 and $\mathrm{S} 66$ in $\mathrm{P} 2 \mathrm{X}_{1}$ ) were affected differently by negatively and positively charged methanethiosulfonate reagents. Furthermore, the reaction of methanethiosulfonate with the I67C mutation was prevented in the presence of $100 \mu \mathrm{M}$ ATP, suggesting an occlusion of the ATP binding site. These data are consistent with our 


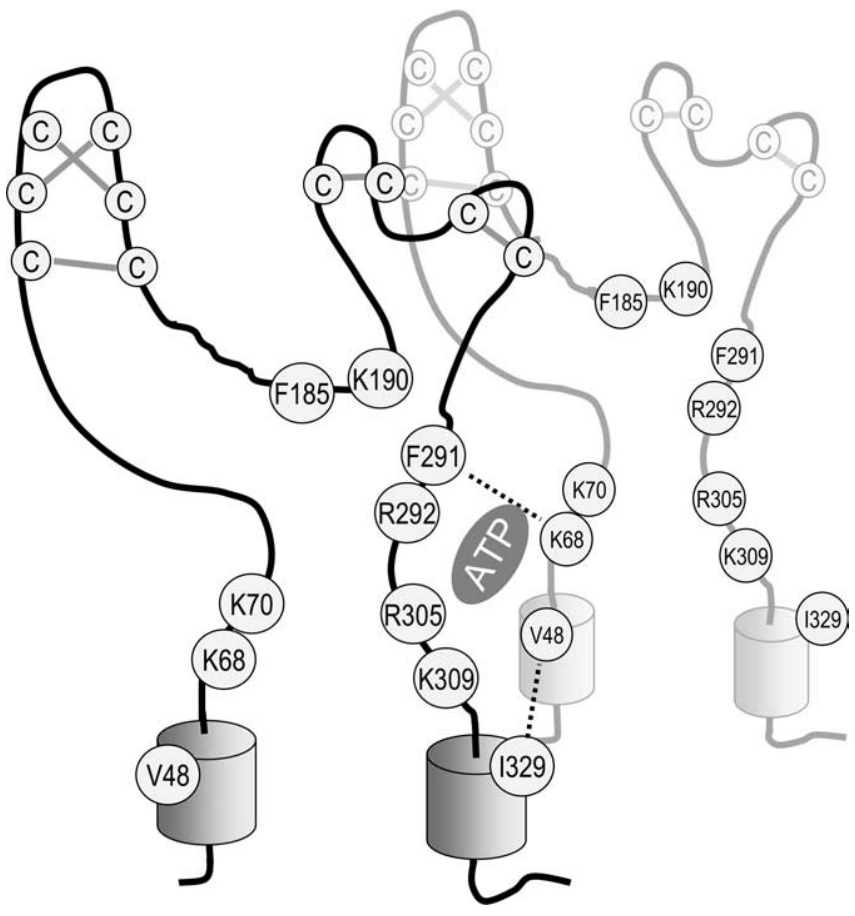

Figure 6. Schematic representation of the proposed localization of the ATP-binding site in $P 2 X_{1}$ Rs. Two P2X subunits are depicted. The localization of the residues mutated in this study in respect to transmembrane regions and the 10 conserved cysteine residues $(C)$ is indicated. On the basis of our results, we suggest that the ATP-binding site is formed at the interface of two neighboring P2X subunits, with residue K68 and the two residues $\mathrm{F} 291$ and K309 being contributed by different subunits. A close proximity of the residues corresponding to V48 (located at the extracellular end of the first transmembrane domain) and 1329 (located at the extracellular end of the second transmembrane domain) from neighboring subunits has been shown previously in $\mathrm{P}_{2} \mathrm{X}_{2} \mathrm{Rs}$ and $\mathrm{P} 2 \mathrm{X}_{2 / 3}$ heteromeric receptors (Jiang et al., 2003). Dashed lines indicate residues that were found to form intersubunit disulfide bridges if replaced by cysteine residues.

biochemical and functional results showing that disulfide bond formation between $\mathrm{K} 68 \mathrm{C}$ and $\mathrm{F} 291 \mathrm{C}$ residues is prevented in the presence of ATP and support their direct involvement in ATP binding. In addition, support for an involvement of the conserved NFR motif (N290-R292) in ATP binding has been presented in a preliminary report on substituted cysteine accessibility mutagenesis in region S286-I329 of the human $\mathrm{P} 2 \mathrm{X}_{1} \mathrm{R}$ (Roberts and Evans, 2006b). Finally, partial and full agonists induce different degrees of conformational changes that lead to channel opening and therefore have also been used to differentiate between mutations that influence gating and binding. Changes in potency of the partial $\mathrm{P} 2 \mathrm{X}_{1}$ agonists BzATP and $\mathrm{Ap}_{5} \mathrm{~A}$ at $\mathrm{P} 2 \mathrm{X}_{1} \mathrm{R}$ mutants have been interpreted in favor of a contribution of K68, F185, F291, R292, and K309 to ATP binding (Roberts and Evans, 2004).

In conclusion, although alternative explanations cannot be excluded, several independent approaches support the hypothesis that all three residues studied here, K68, F291, and K309, are directly involved in ATP binding. In agreement with this interpretation, a model for the ATP-binding domain of the P2XR based on the crystal structure of synapsin II as a structural template proposes that the adenine ring of ATP is complexed between K68 and F291, whereas the K309 residue interacts with its phosphate chain (Roberts et al., 2006).

\section{Intersubunit or intrasubunit agonist-binding site: structural} and pharmacological considerations

Although the disulfide bonding data cannot provide a discrete distance value, the geometric requirements for disulfide forma- tion imply that the respective $\alpha \mathrm{C}$ atoms can converge to a distance of $\sim 4-7 \AA$. This close proximity of K68 and F291 does not necessarily prove that ATP is binding between these two residues (Fig. 6); however, under the most likely assumption that the receptor is formed by a circular and symmetric arrangement of three subunits around the channel pore, the close proximity of K68 and F291 from adjacent subunits implies that both residues are located at opposite ends of a single subunit. Consequently, within the single receptor subunit, these two residues must be located much farther apart, which argues against ATP being complexed between K68 and F291 within a single subunit.

The fact that the combination of K68C and K309C mutations in a single subunit results in nonfunctional receptors, whereas the respective single mutants are functional, indicates that only one of these mutations is tolerated in "functional" ATP binding sites. The functional rescue of double-mutant subunits by heteromerization with nonmutated subunits thus suggests that singlemutated, and hence functional, ATP binding sites can form at the subunit interfaces of double-mutant and nonmutated subunits (Fig. 5B). This further supports the proposal that the ATP binding site is located between subunits. It has to be mentioned, however, that this interpretation relies on the assumption that three ATP molecules must bind to open the P2X channel (Bean et al., 1990; Ding and Sachs, 1999). If binding of only two ATP molecules were sufficient to induce channel opening, heteromers containing one double-mutant and two nonmutated subunits would theoretically also be able to form two functional intrasubunit binding sites within the two nonmutated subunits (Fig. 5C). Because the total number of ATP molecules necessary to induce channel opening is not known, this possibility cannot be excluded. In support of our hypothesis that both subunits contribute to binding-site formation, and consequently binding sites with novel properties are formed at heteromeric interfaces, coexpressed nonmutated and K68C/K309C double-mutant chimeric $\mathrm{P}_{2-1}$ subunits reveal a heteromeric receptor at which the $\mathrm{EC}_{50}$ value for ATP is increased $\left(\mathrm{EC}_{50}=1.7 \mu \mathrm{M}\right)$, as compared with the wt receptor $\left(\mathrm{EC}_{50}=3 \mathrm{nM}\right)$, but is clearly lower than for the homomeric K68C and K309C chimeric mutants ( $\mathrm{EC}_{50}$ values of 204 and $23 \mu \mathrm{M}$, respectively), which should contain only "halffunctional" binding sites. Therefore, we suggest that at least one fully functional binding site is present in the heteromer, which is only the case if two nonmutated chimeras assemble with one double mutant. We exclude the possibility that one ATP molecule is sufficient to open the channel for the following reasons. (1) The Hill-slope of the heteromer generated by coexpression of $\mathrm{K} 68 \mathrm{C} / \mathrm{K} 309 \mathrm{C}$ double-mutant and nonmutated chimeric $\mathrm{P} 2 \mathrm{X}_{2-1}$ subunits is significantly larger than $1\left(n_{\mathrm{h}}=1.5\right)$; and (2) a kinetic model based on single-channel studies by Ding and Sachs (1999) suggests that at least two ATP molecules are required to open the $\mathrm{P} 2 \mathrm{X}_{2} \mathrm{R}$ pore, whereas a model with three ATP molecules fit best to their data.

Our conclusion that the ATP-binding site is formed between adjacent subunits is supported by functional studies at the heteromeric $\mathrm{P} 2 \mathrm{X}_{2 / 3} \mathrm{R}$ (Wilkinson et al., 2006) that were published while this manuscript was in preparation. The authors found that single-mutant (K69A or K308A) $\mathrm{P}_{2} \mathrm{X}_{2}$ subunits but not the respective double-mutant subunits can be fully rescued by coexpression with wt $\mathrm{P} 2 \mathrm{X}_{3}$ subunits. The resulting heteromers display a similar ATP sensitivity as wt $\mathrm{P} 2 \mathrm{X}_{2 / 3}$ heteromers. The respective $\mathrm{P}^{2} \mathrm{X}_{3}$ mutants, on the other hand, could not be rescued by wt $\mathrm{P}_{2} \mathrm{X}_{2}$ subunits. Assuming that the heteromers are formed by two $\mathrm{P}_{2} \mathrm{X}_{3}$ subunits and one $\mathrm{P} 2 \mathrm{X}_{2}$ subunit, they concluded that the lysine residues from the two nonmutated $\mathrm{P} 2 \mathrm{X}_{3}$ subunits interact 
with the single-mutated $\mathrm{P} 2 \mathrm{X}_{2}$ subunits to form two nonmutated and one single-mutated intersubunit binding sites in the heterotrimer.

In conclusion, our study shows a close proximity of two residues of the P2XR that are crucial for ATP potency. Covalent cross-linking of cysteine residues introduced at these positions was shown to have important functional consequences. In the absence of a high-resolution structure or homology model of the $\mathrm{P} 2 \mathrm{XR}$, these findings provide the first direct experimental evidence for an intersubunit ATP binding site of the P2XR and the spatial arrangement of the functionally important K68, F291, and K309 residues. Further exploration of this site will be essential for promoting our understanding of the structure, function, and pharmacology of this receptor family.

\section{References}

Barrera NP, Ormond SJ, Henderson RM, Murrell-Lagnado RD, Edwardson JM (2005) Atomic force microscopy imaging demonstrates that $\mathrm{P}_{2} \mathrm{X}_{2}$ receptors are trimers but that $\mathrm{P} 2 \mathrm{X}_{6}$ receptor subunits do not oligomerize. J Biol Chem 280:10759-10765.

Bean BP, Williams CA, Ceelen PW (1990) ATP-activated channels in rat and bullfrog sensory neurons: current-voltage relation and singlechannel behavior. J Neurosci 10:11-19.

Brejc K, van Dijk WJ, Klaassen RV, Schuurmans M, van Der Oost J, Smit AB, Sixma TK (2001) Crystal structure of an ACh-binding protein reveals the ligand-binding domain of nicotinic receptors. Nature 411:269-276.

Clyne JD, Wang LF, Hume RI (2002) Mutational analysis of the conserved cysteines of the rat $\mathrm{P} 2 \mathrm{X}_{2}$ purinoceptor. J Neurosci 22:3873-3880.

Colquhoun D (1998) Binding, gating, affinity and efficacy: the interpretation of structure-activity relationships for agonists and of the effects of mutating receptors. Br J Pharmacol 125:924-947.

Ding S, Sachs F (1999) Single channel properties of $\mathrm{P}_{2} \mathrm{X}_{2}$ purinoceptors. J Gen Physiol 113:695-720.

Ennion SJ, Evans RJ (2002) Conserved cysteine residues in the extracellular loop of the human $\mathrm{P}_{2} \mathrm{X}_{1}$ receptor form disulfide bonds and are involved in receptor trafficking to the cell surface. Mol Pharmacol 61:303-311.

Ennion S, Hagan S, Evans RJ (2000) The role of positively charged amino

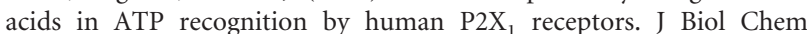
275:35656.

Finger TE, Danilova V, Barrows J, Bartel DL, Vigers AJ, Stone L, Hellekant G, Kinnamon SC (2005) ATP signaling is crucial for communication from taste buds to gustatory nerves. Science 310:1495-1499.

Freist W, Verhey JF, Stühmer W, Gauss DH (1998) ATP binding site of P2X channel proteins: structural similarities with class II aminoacyl-tRNA synthetases. FEBS Lett 434:61-65.

Jiang LH, Rassendren F, Surprenant A, North RA (2000) Identification of amino acid residues contributing to the ATP-binding site of a purinergic P2X receptor. J Biol Chem 275:34190-34196.

Jiang LH, Rassendren F, Spelta V, Surprenant A, North RA (2001) Amino acid residues involved in gating identified in the first membranespanning domain of the rat $\mathrm{P}_{2} \mathrm{X}_{2}$ receptor. J Biol Chem 276:14902-14908.

Jiang LH, Kim M, Spelta V, Bo X, Surprenant A, North RA (2003) Subunit arrangement in P2X receptors. J Neurosci 23:8903-8910.
Mahaut-Smith MP, Ennion SJ, Rolf MG, Evans RJ (2000) ADP is not an agonist at $\mathrm{P}_{2} \mathrm{X}_{1}$ receptors: evidence for separate receptors stimulated by ATP and ADP on human platelets. Br J Pharmacol 131:108-114.

Nagaya N, Tittle RK, Saar N, Dellal SS, Hume RI (2005) An intersubunit zinc binding site in rat $\mathrm{P} 2 \mathrm{X}_{2}$ receptors. J Biol Chem 280:25982-25993.

Nakazawa K, Ojima H, Ishii-Nozawa R, Takeuchi K, Ohno Y (2004) Amino acid substitutions from an indispensable disulfide bond affect $\mathrm{P}_{2} \mathrm{X}_{2}$ receptor activation. Eur J Pharmacol 483:29-35.

Nicke A, Bäumert HG, Rettinger J, Eichele A, Lambrecht G, Mutschler E, Schmalzing G (1998) P2X $\mathrm{X}_{1}$ and $\mathrm{P} 2 \mathrm{X}_{3}$ receptors form stable trimers: a novel structural motif of ligand-gated ion channels. EMBO J 17:3016-3028.

North RA (2002) Molecular physiology of P2X receptors. Physiol Rev 82:1013-1067.

O'Hara PJ, Sheppard PO, Thogersen H, Venezia D, Haldeman BA, McGrane V, Houamed KM, Thomsen C, Gilbert TL, Mulvihill ER (1993) The ligand-binding domain in metabotropic glutamate receptors is related to bacterial periplasmic binding proteins. Neuron 11:41-52.

Rettinger J, Schmalzing G (2003) Activation and desensitization of the recombinant $\mathrm{P}_{2} \mathrm{X}_{1}$ receptor at nanomolar ATP concentrations. J Gen Physiol 121:451-461.

Rettinger J, Schmalzing G (2004) Desensitization masks nanomolar potency of ATP for the $\mathrm{P}_{2} \mathrm{X}_{1}$ receptor. J Biol Chem 279:6426-6433.

Roberts JA, Evans RJ (2004) ATP binding at human P2X receptors: contribution of aromatic and basic amino acids revealed using mutagenesis and partial agonists. J Biol Chem 279:9043-9055.

Roberts JA, Evans RJ (2006a) Contribution of conserved polar glutamine, asparagine and threonine residues and glycosylation to agonist action at human P2X $\mathrm{X}_{1}$ receptors for ATP. J Neurochem 96:843-852.

Roberts J, Evans RJ (2006b) Cysteine scanning mutagenesis of the extracellular loop region 286-329 of the human P2X receptor. Purinergic Signal 2:161.

Roberts JA, Vial C, Digby HR, Agboh KC, Wen H, Atterbury-Thomas A, Evans RJ (2006) Molecular properties of P2X receptors. Pflügers Arch 452:486-500.

Schägger H, Cramer WA, von Jagow G (1994) Analysis of molecular masses and oligomeric states of protein complexes by blue native electrophoresis and isolation of membrane protein complexes by two-dimensional native electrophoresis. Anal Biochem 217:220-230.

Werner P, Seward EP, Buell GN, North RA (1996) Domains of P2X receptors involved in desensitization. Proc Natl Acad Sci USA 93:15485-15490.

Wilkinson WJ, Jiang LH, Surprenant A, North RA (2006) Role of ectodomain lysines in the subunits of the heteromeric $\mathrm{P}_{2} \mathrm{X}_{2 / 3}$ receptor. Mol Pharmacol 70:1159-1163.

Yan Z, Liang Z, Tomic M, Obsil T, Stojilkovic SS (2005) Molecular determinants of the agonist binding domain of a P2X receptor channel. Mol Pharmacol 67:1078-1088.

Yan Z, Liang Z, Obsil T, Stojilkovic SS (2006) Participation of the lys313ile333 sequence of the $\mathrm{P}_{2} \mathrm{X}_{4}$ receptor in agonist binding and transduction of signals to the channel gate. J Biol Chem 281:32649-32659.

Zemkova H, He ML, Koshimizu TA, Stojilkovic SS (2004) Identification of ectodomain regions contributing to gating, deactivation, and resensitization of purinergic P2X receptors. J Neurosci 24:6968-6978. 